\title{
Correlation of Positive and Negative Reciprocity Fails to Confer an Evolutionary Advantage: Phase Transitions to Elementary Strategies
}

\author{
Attila Szolnoki ${ }^{1}$ and Matjaž Perc ${ }^{2}$ \\ ${ }^{1}$ Institute of Technical Physics and Materials Science, Research Centre for Natural Sciences, \\ Hungarian Academy of Sciences, P.O. Box 49, H-1525 Budapest, Hungary \\ ${ }^{2}$ Faculty of Natural Sciences and Mathematics, University of Maribor, Koroška cesta 160, SI-2000 Maribor, Slovenia
}

(Received 18 August 2013; published 27 November 2013)

\begin{abstract}
Economic experiments reveal that humans value cooperation and fairness. Punishing unfair behavior is therefore common, and according to the theory of strong reciprocity, it is also directly related to rewarding cooperative behavior. However, empirical data fail to confirm that positive and negative reciprocity are correlated. Inspired by this disagreement, we determine whether the combined application of reward and punishment is evolutionarily advantageous. We study a spatial public goods game, where in addition to the three elementary strategies of defection, rewarding, and punishment, a fourth strategy that combines the latter two competes for space. We find rich dynamical behavior that gives rise to intricate phase diagrams where continuous and discontinuous phase transitions occur in succession. Indirect territorial competition, spontaneous emergence of cyclic dominance, as well as divergent fluctuations of oscillations that terminate in an absorbing phase are observed. Yet, despite the high complexity of solutions, the combined strategy can survive only in very narrow and unrealistic parameter regions. Elementary strategies, either in pure or mixed phases, are much more common and likely to prevail. Our results highlight the importance of patterns and structure in human cooperation, which should be considered in future experiments.
\end{abstract}

DOI: 10.1103/PhysRevX.3.041021

\section{INTRODUCTION}

Humans have mastered the art of cooperation like no other species [1,2]. Regardless of kinship and individual loss, we work together to achieve feats that are impossible to achieve alone. We have developed a very keen sense of fairness to uphold cooperative behavior in our societies [3,4], and we frequently punish those that do not cooperate in the pursuit of personal benefits and elevated status [5-7]. There also exists evidence that common marmosets and chimpanzees show similar preferences regarding altruism and reward division $[8,9]$, suggesting a long evolutionary history to the human sense of fairness. Although the origins of this behavior are not fully understood, there exists evidence for between-group conflicts [10] and provisioning for someone else's young [11] as viable for igniting the evolution of the remarkable otherregarding abilities of the genus Homo.

Like the origins of cooperative behavior, so, too, do its later development and evolution continue to intrigue and stimulate new research across social and natural sciences [12-16]. Although key mechanisms have been identified that promote the evolution of cooperation [17], there is still disagreement between theory and experiment on many key issues. Two examples have recently attracted notable

Published by the American Physical Society under the terms of the Creative Commons Attribution 3.0 License. Further distribution of this work must maintain attribution to the author(s) and the published article's title, journal citation, and DOI.
Subject Areas: Complex Systems, Interdisciplinary Physics, Statistical Physics

interest. The first concerns network reciprocity [18-21], according to which cooperators are able to exploit the structure of interaction networks to offset inherent evolutionary disadvantages over defectors. Recent large-scale human experiments, however, fail to provide evidence in support of network reciprocity [22]. The second example is of direct relevance for the present work and concerns the strong reciprocity model [23-26]. The latter model postulates that positive and negative reciprocity are directly correlated. In theory, it indeed seems reasonable to assume that rewarding cooperative behavior and punishing unfair behavior are two sides of the same preference for fairness. Yet, recently gathered empirical data suggest otherwise $[27,28]$. In fact, Yamagishi et al. [27] have performed a series of experiments and concluded that there is no correlation between the tendencies to reject unfair offers in the ultimatum game [29] and the tendencies to exhibit prosocial behavior in other games [30,31]. Moreover, the analysis of private household data from the Socio-Economic Panel of the German Institute for Economics Research presented by Egloff et al. [28] has revealed that positive and negative reciprocity vary independently of each other, thus providing a severe challenge to the strong reciprocity model of the evolution of human cooperation. While the rejection of unfair offers, which ought to be seen as equivalent to punishing defection [32], is simply a tacit strategy for avoiding the imposition of an inferior status, the act of cooperating appears to have an altogether different motivational background. 
The described disagreement between the strong reciprocity model and empirical data invites an interdisciplinary approach, which promises to shed light on the subject from a different perspective. In the present paper, we therefore apply evolutionary game theory [33-37] and methods of statistical physics [38,39] to determine whether there are evolutionary advantages to be gained by adopting a strategy that punishes defectors as well as rewards cooperators, as opposed to doing just one or the other. While the elementary strategies of rewarding and punishment have received ample attention in the recent past [40-45], little is known about their combined effectiveness. To amend this knowledge gap, we propose and study a modified spatial public goods game [46,47], where defectors compete with cooperators that punish defectors, reward other cooperators, as well as do both. We intentionally leave out cooperators that neither reward nor punish in order to avoid the second-order freeriding problem $[48,49]$ and to thus be able to focus solely on the effectiveness of the combined strategy against the three elementary strategies of defection, rewarding, and punishment.

As we will show in what follows, although the spatiotemporal dynamics of the evolutionary game is very complex and interesting from the physics point of view, there exist only narrow and realistically unlikely parameter regions where the combined strategy is able to survive. Given the lack of notable evolutionary advantages of correlating positive and negative reciprocity, the outcome of the experiments by Yamagishi et al. [27] and Egloff et al. [28] can thus be better understood, although the complexity of solutions also lends some support to the strong reciprocity hypothesis as being viable at least under certain special circumstances. We will present compelling evidence to support these conclusions in Sec. III, while in the next section, we first describe the studied spatial public goods game and the methods in more detail.

\section{PUBLIC GOODS GAME WITH POSITIVE AND NEGATIVE RECIPROCITY}

As a frequently used paradigm of social conflicts and human cooperation, the public goods game is staged on a square lattice with periodic boundary conditions where $L^{2}$ players are arranged into overlapping groups of size $G=5$ such that everyone is connected to its $G-1$ nearest neighbors. Accordingly, each individual belongs to $g=1, \ldots, G$ different groups. The square lattice is the simplest of networks that allows us to go beyond the unrealistic well-mixed population assumption, and as such, it allows us to take into account the fact that the interactions among humans are inherently structured rather than random. By using the square lattice, we also continue a long-standing history that began with the work of Nowak and May [18], who were the first to show that the most striking differences in the outcome of an evolutionary game emerge when the assumption of a well-mixed population is abandoned for the usage of a structured population. Many have since followed the same practice [46,50,51] (for a review, see Ref. [14]), and there exists ample evidence in support of the claim that, especially for games that are governed by group interactions [47,52], using the square lattice suffices to reveal all the feasible evolutionary outcomes, and also that these outcomes are qualitatively independent of the interaction structure.

Initially, each player on site $x$ is designated either as a defector $\left(s_{x}=D\right)$, a cooperator that punishes defectors $\left(s_{x}=P\right)$, a cooperator that rewards other cooperators $\left(s_{x}=R\right)$, or a cooperator that both punishes defectors as well as rewards other cooperators $\left(s_{x}=B\right)$ with equal probability. All three cooperative strategies $(P, R$, and $B)$ contribute a fixed amount (here considered to be equal to 1 without loss of generality) to the public good, while defectors contribute nothing. The sum of all contributions in each group is multiplied by the synergy factor $r$, and the resulting public goods are distributed equally among all the group members, irrespective of their strategies. In addition, a defector suffers a fine $\beta /(G-1)$ from each punisher $(P$ or $B)$ within the interaction neighborhood, which in turn requires the punisher to bear the cost $\gamma /(G-1)$ on each defecting individual in the group. A defector thus suffers the maximal fine $\beta$ if it is surrounded solely by punishers, while a lonely punisher bears the largest cost $\gamma$ if it is surrounded solely by defectors. Similarly, every cooperator is given the reward $\beta /(G-1)$ from every $R$ and $B$ player within the group, while each of them has to bear the cost of rewarding $\gamma /(G-1)$ for every cooperator that is rewarded. As a technical comment, we note that the application of payoffs normalized by $G-1$ enables relevant comparisons with the evolutionary outcomes on other interaction networks where players might differ in their degree and group size. Moreover, we use an equally strong fine and reward at the same cost, technically the same pair of $(\beta, \gamma)$ values for reward and punishment, which ensures a fair evaluation of the evolutionary advantage of both strategies. Decoupling these parameters, for example, by administering high fines and low rewards at the same cost to both punishers and those that reward, would confer an unfair advantage to punishment because it would then be relatively less costly than rewarding. Since giving equal chances for success is of paramount importance for assessing evolutionary viability, we do not decouple $\beta$ and $\gamma$ for reward and punishment, and we also award limitless resources to all competing strategies.

In agreement with the described rules of the game, the payoff values of the four competing strategies obtained from each group $g$ are thus 


$$
\begin{aligned}
& \Pi_{D}^{g}=r\left(N_{P}+N_{R}+N_{B}\right) / G-\beta\left(N_{P}+N_{B}\right) /(G-1), \\
& \Pi_{P}^{g}=r\left(N_{P}+N_{R}+N_{B}+1\right) / G-\gamma N_{D} /(G-1)+\beta\left(N_{R}+N_{B}\right) /(G-1), \\
& \Pi_{R}^{g}=r\left(N_{P}+N_{R}+N_{B}+1\right) / G-\gamma\left(N_{P}+N_{R}+N_{B}\right) /(G-1)+\beta\left(N_{R}+N_{B}\right) /(G-1), \\
& \Pi_{B}^{g}=r\left(N_{P}+N_{R}+N_{B}+1\right) / G-\gamma+\beta\left(N_{R}+N_{B}\right) /(G-1),
\end{aligned}
$$

where $N_{s_{x}}$ denotes the number of other players with strategy $s_{x}$ in the group.

Monte Carlo simulations of the public goods game are carried out comprising the following elementary steps. A randomly selected player $x$ plays the public goods game with its $G-1$ partners as a member of all the $g=1, \ldots, G$ groups, whereby its overall payoff $\Pi_{s_{x}}$ is thus the sum of all the payoffs $\Pi_{s_{x}}^{g}$ acquired in each individual group. Next, player $x$ chooses one of its nearest neighbors at random, and the chosen coplayer $y$ also acquires its payoff $\Pi_{s_{y}}$ in the same way. Finally, player $x$ enforces its strategy $s_{x}$ onto player $y$ with a probability given by the Fermi function $w\left(s_{x} \rightarrow s_{y}\right)=$ $1 /\left\{1+\exp \left[\left(\Pi_{s_{y}}-\Pi_{s_{x}}\right) / K\right]\right\}$, where $K=0.5$ quantifies the uncertainty by strategy adoptions [47], implying that the strategies of better-performing players are readily adopted, although it is not impossible to adopt the strategy of a player that performs worse. Such errors in decision making can be attributed to mistakes and external influences that adversely affect the evaluation of the opponent. Each Monte Carlo step (MCS) gives a chance for every player to enforce its strategy onto one of its neighbors once, on average.

The average fractions of defectors $\left(\rho_{D}\right)$, cooperators that punish $\left(\rho_{P}\right)$, cooperators that reward $\left(\rho_{R}\right)$, and cooperators that do both $\left(\rho_{B}\right)$ on the square lattice are determined in the stationary state after a sufficiently long relaxation time. Depending on the actual conditions (proximity to phase transition points and the typical size of emerging spatial patterns), the linear system size is varied from $L=400$ to 7200 and the relaxation time is varied from $10^{4}$ to $10^{5} \mathrm{MCS}$ to ensure that the statistical error is comparable with the line thickness in the figures. We note that the random initial state may not necessarily yield a relaxation to the most stable solution of the game even at such a large system size $(L=7200)$. To verify the stability of different solutions, we have therefore applied prepared initial states (see Fig. 10 in Ref. [53]) and we have followed the same procedure as described previously in Ref. [54]. Next, we proceed with presenting the main results.

\section{RESULTS}

Systematic Monte Carlo simulations are performed to reveal phase diagrams for two representative values of the synergy factor $r$. In the absence of reward and punishment, cooperators survive only if $r>3.74$, and they are able to defeat defectors completely for $r>5.49$ [47]. Taking these thresholds as benchmark values, we focus on $r=4.5$ and $r=2.5$. For $r=4.5$, cooperators are able to coexist with defectors without support from additional strategies, solely on the basis of network reciprocity. This value of $r$ thus yields lenient conditions for the evolution of public cooperation. For $r=2.5$, on the other hand, cooperators are unable to survive in the absence of reward and punishment. This value of $r$ thus yields adverse conditions for cooperative behavior to prevail. For both values of $r$, we determine the stationary fractions of strategies when varying the reward or fine $\beta$ and the cost $\gamma$. The transition points and the type of phase transitions are identified from Monte Carlo data collected with a sufficiently high accuracy (and frequency) in the close vicinity of the transition points. Finally, the phase boundaries, separating different stable solutions, are plotted in the full $\beta-\gamma$ phase diagrams. The obtained quantitative results are discussed in detail in the following two subsections.

\section{A. Synergy factor $r=4.5$}

The phase diagram depicted in Fig. 1 suggests that at such a high value of $r$, the far more effective action to outperform defectors is punishment rather than rewarding. The pure (or absorbing because the applied dynamical rule

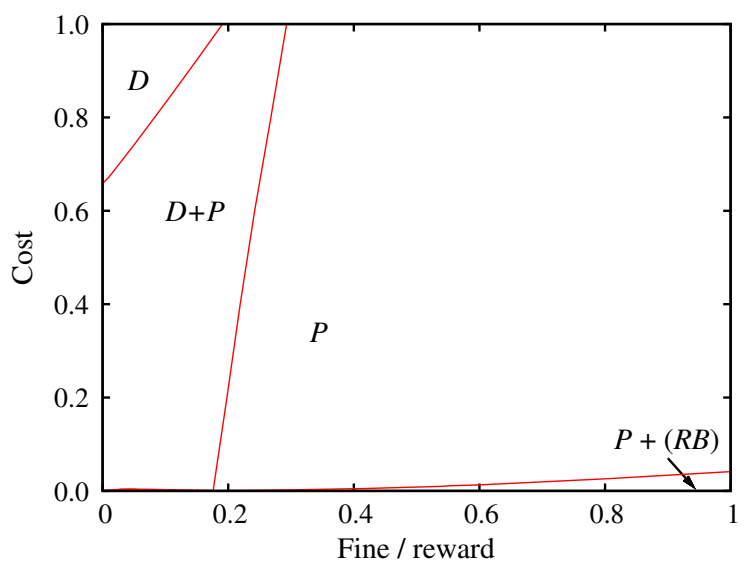

FIG. 1. Full $\beta-\gamma$ phase diagram, as obtained for $r=4.5$. Solid red lines denote continuous phase transitions. If defectors die out, strategies $R$ and $B$ become equivalent (see the main text for details), hence the $(R B)$ notation in the lower right corner of the parameter plane. The vertical resolution hides the intricate structure of the phase diagram for very low values of $\gamma$, which we therefore show separately in Fig. 2. 


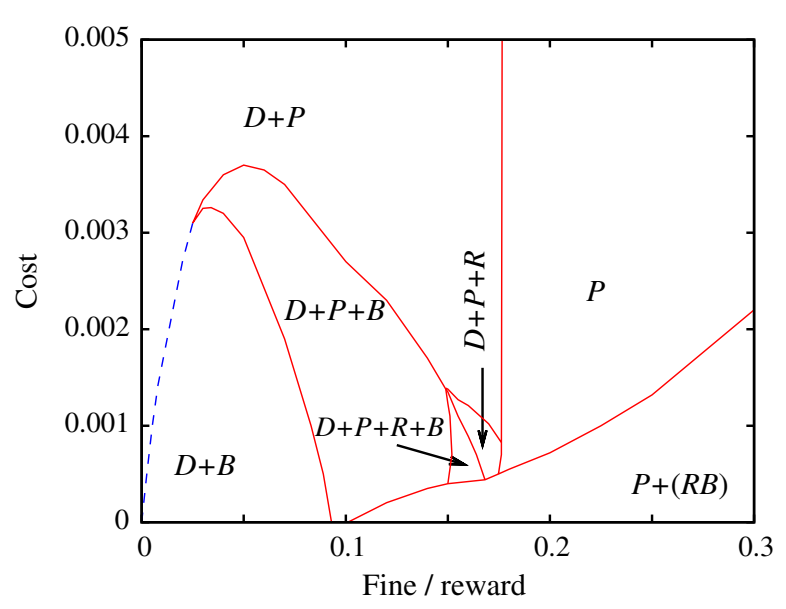

FIG. 2. Enlarged part of the full $\beta-\gamma$ phase diagram depicted in Fig. 1, zooming in on the very small (approximately $10^{3}$ smaller than $\beta$ ) values of $\gamma$. Solid red lines denote continuous phase transitions, while the dashed blue line denotes discontinuous phase transitions. Discontinuous phase transitions are due to indirect territorial competition between strategies $P$ and $B$, which compete independently against the defectors. A representative cross section of the phase diagrams is presented in Fig. 3.

leaves the phase unchanged once the system arrives there) $D$ phase in the upper left corner of the $\beta-\gamma$ plane first gives way to the mixed $D+P$ phase and subsequently to the pure $P$ phase as the fine (cost) increases (decreases). Only if the cost is negligible and the fine or reward is large are rewarding strategies able to survive. In this case, defectors die out very soon, and from there on, strategies $R$ and $B$ become equivalent since there is nobody left to punish. For the same reason, strategy $P$ transforms to that of ordinary cooperation. Accordingly, strategies $R$ and $B$ are able to coexist alongside strategy $P$ as long as the cost of rewarding is sufficiently small enough to offset the second-order free riding (because $P$ does not contribute to rewarding other cooperators). The phase is denoted appropriately as $P+(R B)$ in the lower right corner of Fig. 1.

Yet, Fig. 1 fails to convey the full story behind the depicted phase diagram. For very low values of $\gamma$ (approximately $10^{3}$ smaller than $\beta$ ), the studied spatial public goods game reveals its true potential to yield rich dynamical behavior that gives rise to a truly intricate phase diagram. As can be observed in Fig. 2, no fewer than seven successive phase transitions can occur upon varying a single parameter (increasing $\beta$ at a fixed value of $\gamma$ ). In addition to the pure $P$ phase, we can observe two-strategy $D+P, D+B$, and $P+(R B)$ (note that here, $R$ and $B$ are equivalent strategies) phases, three-strategy $D+P+B$ and $D+P+R$ phases, and even the four-strategy $D+$ $P+R+B$ phase. While the majority of phase transitions is continuous, the $D+P \rightarrow D+B$ phase transition is discontinuous because of an indirect territorial competition (see Refs. [55,56] for further examples of this

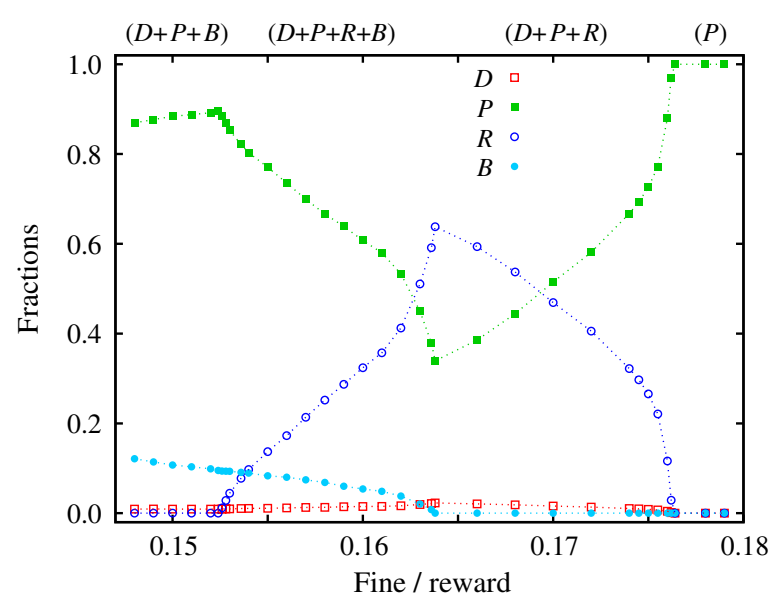

FIG. 3. Cross section of the phase diagram depicted in Fig. 2, as obtained for $\gamma=0.0007$. Depicted are stationary fractions of the four competing strategies in dependence on $\beta$. Stable solutions are denoted along the top axis. Unlike the $D+P \rightarrow D+B$ phase transition, denoted by a dashed blue line in Fig. 2, in this cross section, all phase transitions are continuous.

phenomenon) between strategies $P$ and $B$. The two compete independently against the defectors, and the victor is determined by whoever is more effective. The nature of the other phase transitions is illustrated quantitatively in Fig. 3, which shows a cross section across $\beta$ for the most interesting value of $\gamma$.

Despite the complexity of solutions, the relevance of the presented results for the main question addressed in this study is quickly revealed. The dashed blue line in Fig. 2, marking the discontinuous $D+P \rightarrow D+B$ phase transition, conveys directly that the combined strategy $B$ is more effective than the elementary strategy $P$ only if $\beta$ increases [if conditions for rewarding and sanctioning become more lenient (the two actions become less costly)], and this shift in effectiveness happens only when the costs are already negligible (approximately $10^{3}$ smaller than the administered rewards and fines). Accordingly, we conclude that, at least for high values of the synergy factor $r$, there are no notable evolutionary advantages associated with correlating positive (rewarding) and negative (punishment) reciprocity in a single strategy. This conclusion agrees with the empirical data presented by Yamagishi et al. [27] and Egloff et al. [28], who failed to observe the same correlation in human experiments. On the other hand, it should not be overlooked that the combined strategy $B$ is viable and that it does convey some advantages (albeit in very narrow and rather unrealistic parameter regions), which thus also lends some support to the strong reciprocity model [24].

To demonstrate just how the combined strategy $B$ may survive, we show in Fig. 4 a series of snapshots from a prepared initial state (applied solely to allow the usage of a relatively small system size), which eventually evolves 

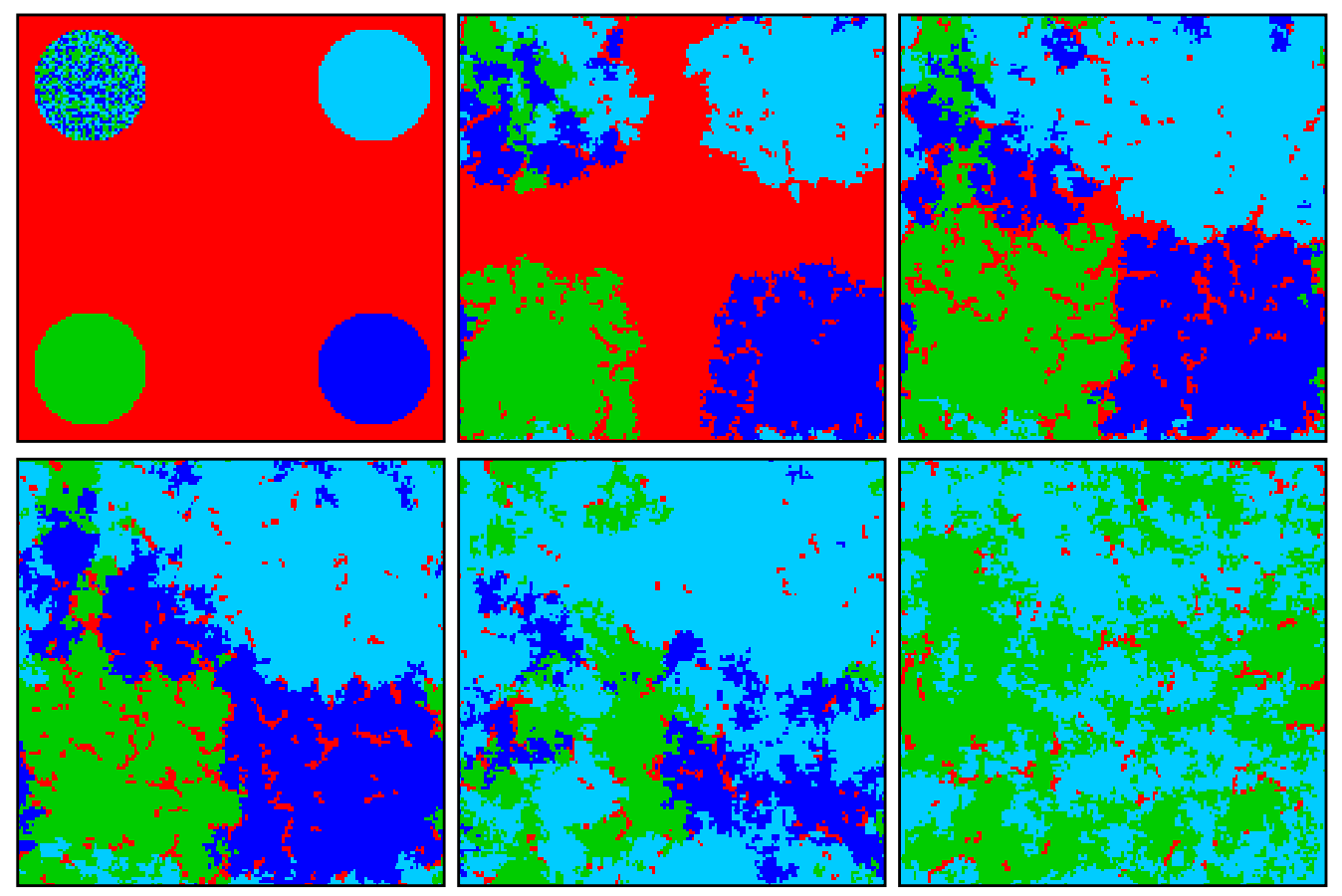

FIG. 4. Snapshots of the square lattice, showing a characteristic evolution from a prepared initial state, as obtained for $\gamma=0.0015, \beta=0.1$, and $r=4.5$. Strategies $D, P, R$, and $B$ are depicted in red, green, dark blue, and light blue, respectively. (The same colors are used in Fig. 3.) Time runs from the top left panel toward the bottom right panel at 0, 100, 210, 300, 1130, and 8700 Monte Carlo steps, respectively. At $0 \mathrm{MCS}$, the game is initiated from a prepared initial state (the upper left domain is a mixture of strategies $P, R$, and $B)$ to allow the usage of a very small system size $(L=150)$ that still allows us to infer strategy configurations in sufficient detail. At $100 \mathrm{MCS}, D$ (red) percolates slightly into green, light blue, and dark blue clusters, indicating that all three cooperative strategies could form a two-strategy phase with defectors. At 210 MCS, the borders of the three mentioned two-strategy phases $(D+P, D+R$, and $D+B)$ meet, and at $300 \mathrm{MCS}$, it can be observed that strategy $B$ (light blue) is capable of invading into the $D+P$ phase while strategy $R$ is unable to do the same. While $R$ and $B$ are neutral in the absence of $D$, in direct competition against $D$, strategy $B$ is more effective and thus continues to crowd out strategy $R$, as can be inferred at 1130 MCS. Interestingly, strategy $P$ has a small advantage over strategy $B$ because strategy $P$ can spare the cost of rewarding. Yet, this advantage is sufficient for $P$ to survive in the bulk of strategy $B$. The last panel, taken at 8700 MCS, depicts a typical stationary pattern where strategies $D, P$, and $B$ coexist to form the $D+P+B$ phase.

toward the three-strategy $D+P+B$ phase. Based on this example, it could be argued that adopting strategy $B$ does in fact confer an advantage over strategy $R$, which succumbs to the evolutionary pressure stemming from the three surviving strategies. However, as can be observed at a glance from the depicted phase diagrams presented in Figs. 1 and 2, the evolutionary advantage of strategy $B$ over strategy $R$ is limited to a very narrow and specific parameter range, which is practically invisible at normal resolution (see Fig. 1). In addition, we emphasize that strategy $B$ is slightly less effective than strategy $P$ (see Fig. 3 for the stationary fractions of the two strategies). Thus, although the combined strategy $B$ might appear as a good choice in some of the parameter regions within Fig. 2, it is still second to the elementary strategy $P$ that adopts solely punishment.

\section{B. Synergy factor $r=\mathbf{2 . 5}$}

If the conditions for the evolution of public cooperation become harsh, as is the case for $r=2.5$, the relations between the competing strategies change quite significantly. The phase diagram presented in Fig. 5 reveals that, besides the expected extension of the pure $D$ phase, the parameter region where strategy $B$ can survive also becomes larger. Furthermore, there is a significant change in the nature of phase transitions. Unlike at $r=$ 4.5 (see Fig. 1), here discontinuous phase transitions dominate, which has to do with the spontaneous emergence of cyclic dominance [57-60] between strategies $D, P$, and $B$. In particular, within the three-strategy $D+$ $P+B$ phase, strategy $D$ outperforms strategy $P$, strategy $P$ outperforms strategy $B$, while strategy $B$ again outperforms strategy $D$. It is important to note that at $r=4.5$, the stability of neither the three-strategy phases nor the four-strategy phase has been due to cyclic dominance. Instead, as Fig. 4 illustrates, there the stability was warranted by the stable coexistence of the strategies, rather than by oscillations that are brought about by cyclic dominance.

As was frequently the case before $[53,54,61,62]$, here, too, the spontaneous emergence of cyclic dominance 


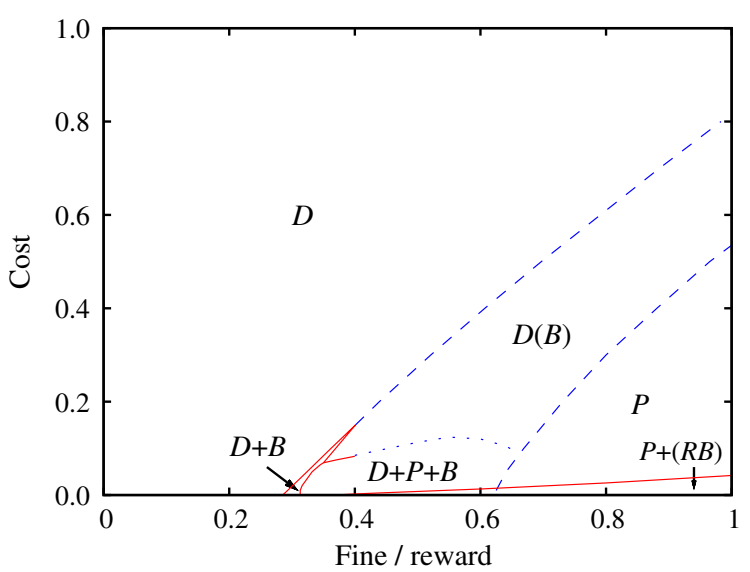

FIG. 5. Full $\beta-\gamma$ phase diagram, as obtained for $r=2.5$. Solid red lines denote continuous phase transitions, while dashed blue lines denote discontinuous phase transitions. More precisely, the dashed blue line between phases $D$ and $D(B)$ indicates that there would be a discontinuous phase transition if only $D$ and $B$ strategies were initially present in the system. (In other words, the frontier of dominance between strategies $D$ and $B$ is delineated.) The same holds for the dashed blue line separating phases $D(B)$ and $P$. The three-strategy $D+P+B$ phase is separated with a dotted blue line to emphasize that there are two very different ways in which this solution can give way to the $D(B)$ phase. In particular, at smaller fines, the transition is continuous because the average fraction of strategy $P$ gradually decays to zero (see Fig. 6 for a quantitative insight). At larger fines, however, the averages of all three strategies remain finite, but the amplitude of oscillations diverges regardless of the system size (see Fig. 8 for details), which ultimately results in an abrupt termination of cyclic dominance between the three strategies (see Fig. 7). The notation of the $P+(R B)$ phase at the lower right corner of the phase diagram has the same meaning as described in the caption of Fig. 1.

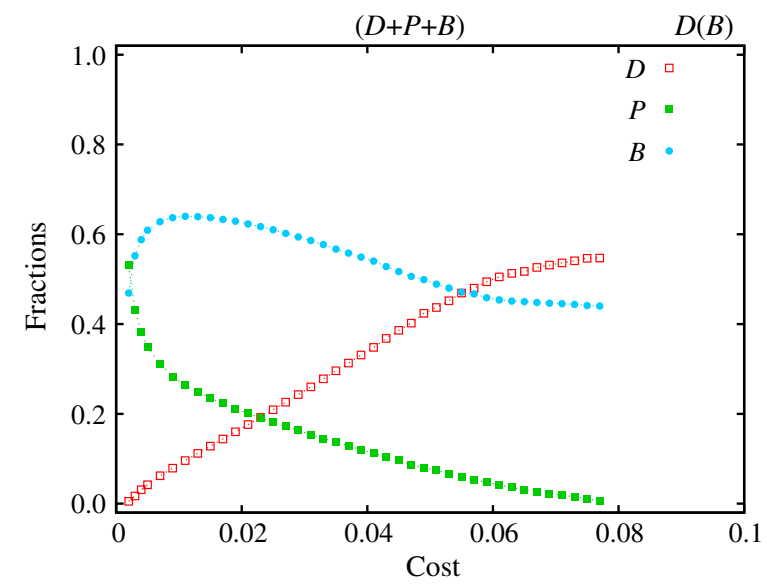

FIG. 6. Cross section of the phase diagram depicted in Fig. 5, as obtained for $\beta=0.37$. Depicted are stationary fractions of the four competing strategies in dependence on $\gamma$. Stable solutions are denoted along the top axis. In this cross section, the $D+P+B \rightarrow D(B)$ phase transition is continuous because the average fraction of strategy $P$ gradually decays to zero as $\gamma$ increases. brings with it fascinating dynamical processes that are driven by pattern formation, by means of which the phase may terminate. Figures 6 and 7 feature two characteristic cross sections of the phase diagram presented in Fig. 5, which reveal two qualitatively very different ways for the $D+P+B$ cyclic dominance phase to give way to the $D(B)$ phase. [Here, $D(B)$ indicates that either a pure $D$ or a pure $B$ phase can be the final state if starting from random initial conditions.] The process depicted in Fig. 6 is relatively straightforward. Here, the average fractions of strategies $P$ and $B$ decay because of the increasing cost $\gamma$, which ultimately results in the vanishing average value of the fraction of strategy $P$. The closed cycle of dominance is therefore interrupted, and the $D+P+B$ phase terminates.

The situation for $\beta=0.55$ is much more peculiar and interesting. As results presented in Fig. 7 demonstrate, here the average values of all three strategies remain finite. Hence, the termination of the $D+P+B$ phase must have a different origin than at $\beta=0.37$ presented in Fig. 6. In fact, for $\beta=0.55$, it is the amplitude of oscillations that increases with increasing values of $\gamma$. And, it is the increase in the amplitude that ultimately results in a uniform absorbing phase regardless of the system size. At this point, it is crucial to emphasize that the increase of the amplitude of oscillation is not a finitesize effect. Although, in spatial systems with cyclic dominance, it is typical to observe oscillations with increasingly smaller amplitude as the system size is increased, this decrease in amplitude does not occur in the present case. To demonstrate the lack of decreasing amplitude as the system increases, we measure the fluctuations in the stationary state according to

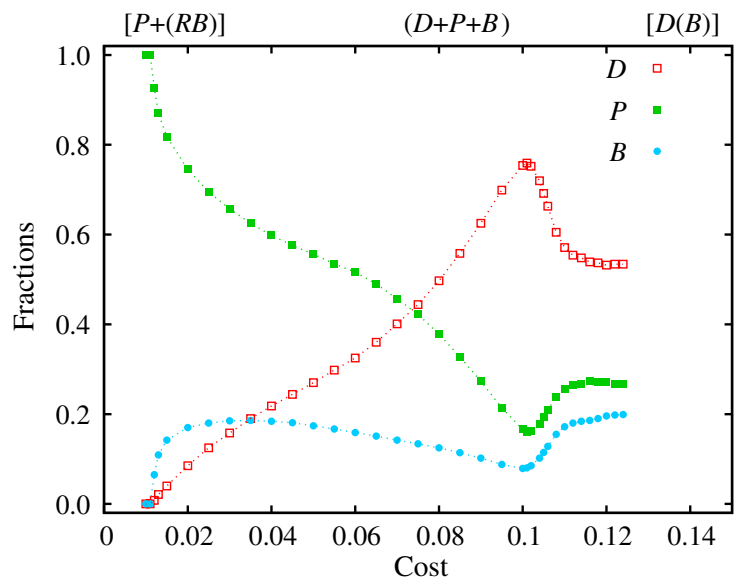

FIG. 7. Cross section of the phase diagram depicted in Fig. 5, as obtained for $\beta=0.55$. Depicted are stationary fractions of the four competing strategies in dependence on $\gamma$. Stable solutions are denoted along the top axis. Unlike in Fig. 6, here the $D+$ $P+B \rightarrow D(B)$ phase transition is discontinuous because the amplitude of oscillations diverges independently of the system size (see Fig. 8 for details) as $\gamma$ increases. 


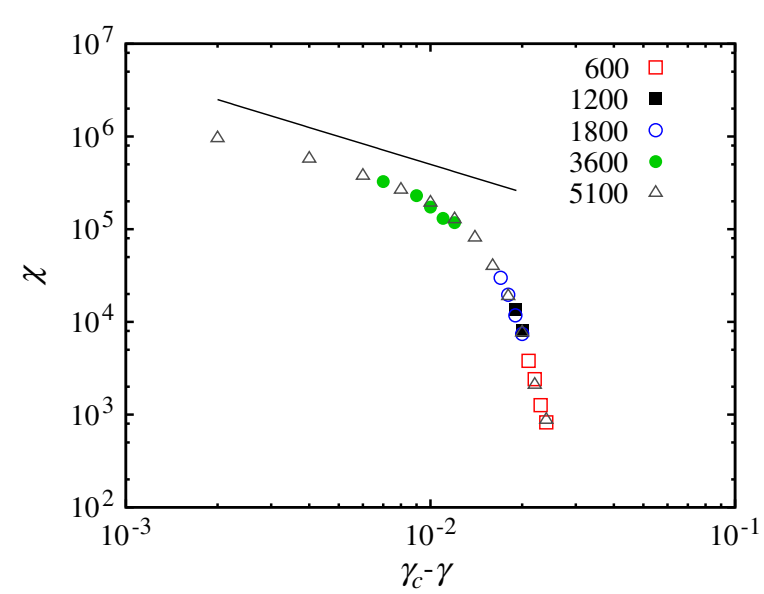

FIG. 8. Fluctuations of the amplitude of oscillations $\chi$ in dependence on the vicinity to the critical value of the cost $\gamma_{c}=0.1242(6)$ for $\beta=0.55$ and different system sizes, as indicated in the legend. The slope of the power-law exponent (solid line) is 1 , indicating divergent fluctuation as $\gamma$ approaches the critical value.

$$
\chi=\frac{L^{2}}{M} \sum_{t_{i}=1}^{M}\left\langle\left[\rho_{D}\left(t_{i}\right)-\overline{\rho_{D}}\right]^{2}\right\rangle,
$$

where $\overline{\rho_{D}}$ is the average value of the fraction of defectors. (A similar quantity can be calculated for the other strategies as well.) As Fig. 8 shows, the scaled quantity $\chi$ is size independent, thus indicating a divergent fluctuation as $\gamma$ approaches the critical value. The three-strategy $D+P+B$ phase is therefore unable to exist beyond this value, despite the fact that the average fractions of all three strategies are far from zero. Instead, the phase terminates via a discontinuous phase transition toward the $D(B)$ phase, as depicted in Fig. 5. Notably, within the $D(B)$ phase, either the pure $D$ or the pure $B$ phase can be the final state, depending on which strategy dies out first.

With this analysis, however, we have not yet covered all the details of the phase diagram presented in Fig. 5. In addition, there are, namely, the same pure $P$ and twostrategy $P+(R B)$ phases observable that we have already reported above for $r=4.5$ (see Fig. 1), only at $r=2.5$, they are shifted further toward higher values of $\beta$. This shift is understandable, given that the lesser support for public cooperation due to a lower value of the synergy factor needs to be offset by higher fines and rewards. Moreover, we must not overlook the existence, albeit a very subtle one, of the two-strategy $D+B$ phase, the emergence of which is quantitatively described in the cross section presented in Fig. 9. The $D+P$ phase is the only stable solution where the combined strategy $B$ coexists with defectors and where thus the correlation of negative and positive reciprocity truly outperforms elementary strategies $P$ and $R$. As in all the previously outlined cases, however, in this case, too, this

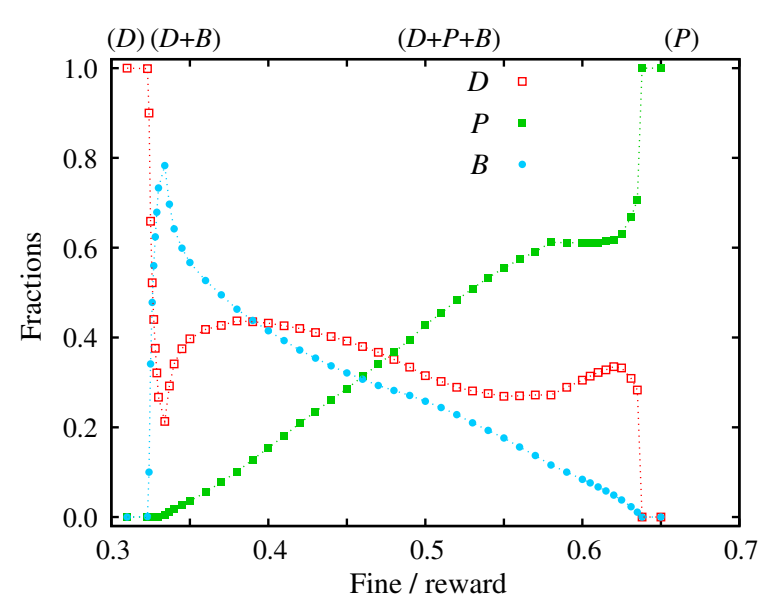

FIG. 9. Cross section of the phase diagram depicted in Fig. 5, as obtained for $\gamma=0.05$. Depicted are stationary fractions of the four competing strategies in dependence on $\beta$. Stable solutions are denoted along the top axis. In this cross section, the pure $D$ phase first gives way to a very narrow interval where the two-strategy $D+B$ phase precedes the spontaneous emergence of cyclic dominance between strategies $D, P$, and $B$. The last (from left to right) $D+P+B \rightarrow P$ phase transition is qualitatively similar to the $D+P+B \rightarrow D(B)$ phase transition depicted in Fig. 6, only here it is the fraction of strategy $B$ that decays to zero as $\beta$ increases, and which thus interrupts the closed cycle of dominance. (In Fig. 6, it is the fraction of strategy $P$ as $\gamma$ increases that has the same effect.)

advantage is minute and limited to a very narrow region in the phase diagram.

In general, the harsher conditions for the evolution of public cooperation lend more support for the combined strategy to survive, as indeed the regions on the $\beta-\gamma$ parameter plane where $B$ can prevail become quite extensive at smaller values of the synergy factor $r$. This expansion of the $B$ phase extends the credibility of the strong reciprocity model, and it indicates that, if they manifest at all, the evolutionary advantages of correlated positive and negative reciprocity ought to manifest more clearly under extreme adversity. In future experiments, it may thus be worthwhile to work toward such conditions if the goal is to possibly discern some more actual advantages of correlated reciprocities and thus to further support the assumptions of the strong reciprocity theory with empirical data. A warning to end the presentation of results is, however, in order. As the series of final snapshots presented in Fig. 10 clearly demonstrates (and to no lesser extent also the series of snapshots presented in Fig. 4), conditions for pattern formation and complex strategic configurations need to be given for the subtle solutions, here identified by means of extensive and systematic Monte Carlo simulations, to emerge and be stable. Such conditions appear to be very difficult to achieve in experiments with humans, which is why efforts toward large-scale implementations, as recently 

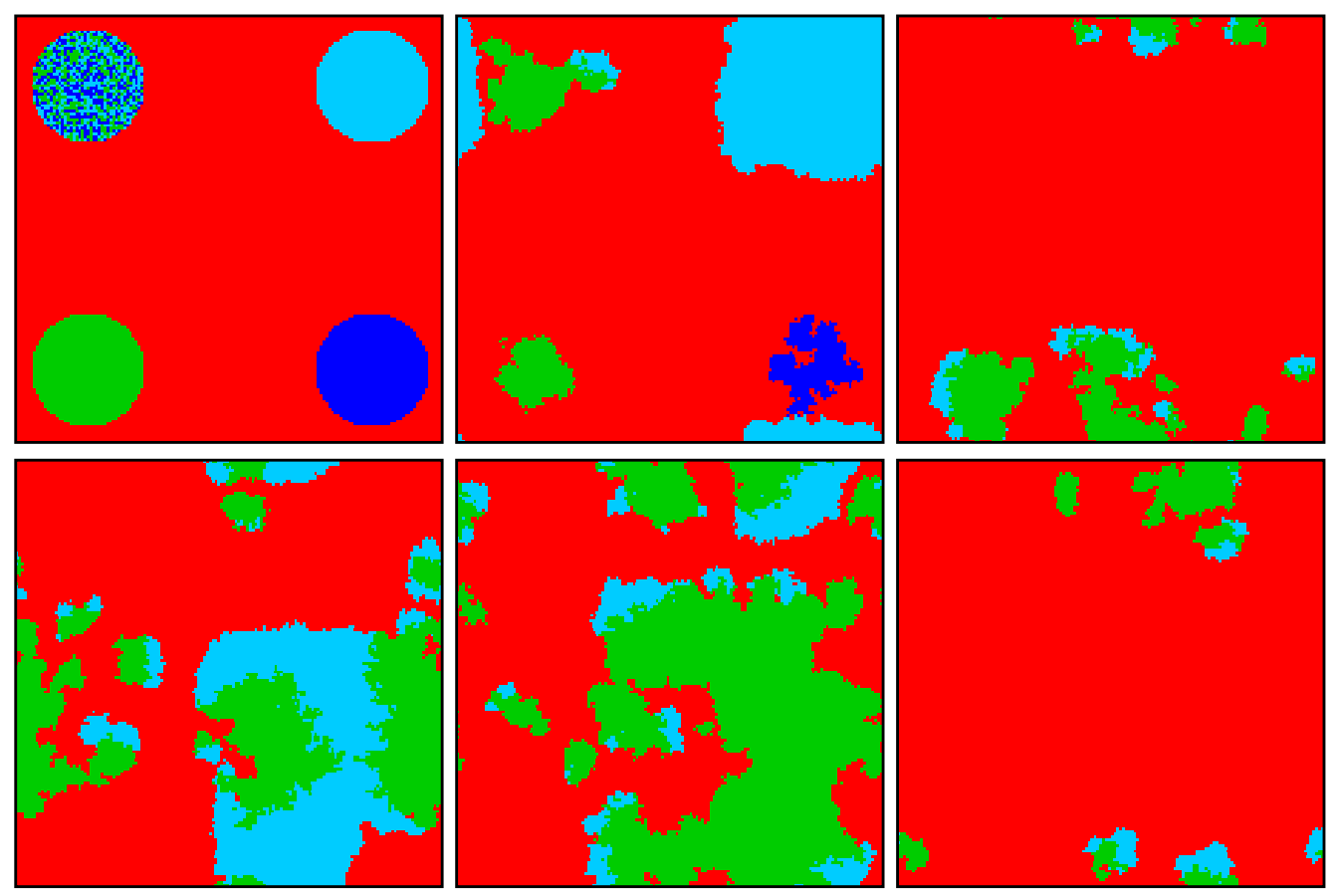

FIG. 10. Snapshots of the square lattice, showing a characteristic evolution from a prepared initial state, as obtained for $\gamma=0.1$, $\beta=0.55$, and $r=2.5$. Strategies $D, P, R$, and $B$ are encoded with the same color as used in Fig. 4. Time runs from the top left panel toward the bottom right panel at 0, 100, 1800, 4100, 4240, and 4610 Monte Carlo steps, respectively. At 0 MCS, the game is initiated from the same prepared initial state, and for the same reason, as described in the caption of Fig. 4. At 100 MCS, it can be observed that strategies $P$ (green) and $R$ (dark blue) are both weaker than strategy $D$ (red), and accordingly, their isolated islands shrink. Conversely, the combined strategy $B$ is more effective when competing alone against the defectors, and thus the light blue island grows. Moreover, in the absence of defectors, strategy $P$ can exploit rewarding strategies and spread fast in the bulk of the mixed domain (upper left circle). It is also worth pointing out that the $B$ domain would grow endlessly in the sea of defectors if it did not meet the elementary strategy $P$ that is able to exploit it. At $1800 \mathrm{MCS}$, the final solution is practically formed, and from here on, traveling waves dominate the spatial grid. At $4100 \mathrm{MCS}$, it can be observed that strategy $B$ can spread toward strategy $D$, and based on this invasion, strategy $B$ may control a significant portion of the lattice for a short period of time. At $4240 \mathrm{MCS}$, however, strategy $P$ can easily invade the bulk of the $B$ domain, but in the absence of this domain, strategy $P$ itself becomes vulnerable against the defectors. This cycle of dominance is repeated from $4610 \mathrm{MCS}$ onward, which is a very similar configuration as the one at $1800 \mathrm{MCS}$. Naturally, the oscillations become more intense as we approach the edge of the $D+P+B$ phase in Fig. 5, and the evolution can easily terminate in an absorbing phase if the system size is not sufficiently large.

reported in Refs. [22,63], are very encouraging and certainly worth developing further in the future.

\section{DISCUSSION}

Our goal in the present paper was to determine whether there are evolutionary advantages associated with correlating positive and negative reciprocity in a single strategy, as opposed to adopting solely reward or punishment as an elementary strategy. Systematic Monte Carlo simulations have revealed that, regardless of the synergy factor governing the public goods game, elementary strategies, and punishment in particular, are, in general, significantly more effective in deterring defection than the combined strategy. Although there exist narrow and rather unrealistic parameter regions where the correlation of positive and negative reciprocity can outperform a particular elementary strategy, these advantages are highly unlikely to play a role in human experiments, and they also frequently come second to the evolutionary success that is warranted by punishment alone under the same conditions. The presented results thus lend support to the empirical data published in Refs. [27,28], which fail to support the central assumption of the strong reciprocity model that negative and positive reciprocity are correlated.

The studied four-strategy spatial public goods game gives rise to fascinating evolutionary outcomes that are separated by continuous and discontinuous phase transitions. We have demonstrated, for example, that indirect territorial competition may lend some credibility to the combined strategy, as the latter is sometimes more effective against the defectors than solely rewarding. In special parameter regions, the combination of positive and negative reciprocity can thus crowd out cooperators that reward other cooperators. Under the same conditions, however, cooperators that punish but do not reward can be more effective still, so overall, it is difficult to argue in favor of choosing the combined strategy over an elementary 
one. Moreover, stationary solutions that are governed by indirect territorial competition terminate suddenly via discontinuous phase transitions, and accordingly, they are difficult to identify and are unlikely to seriously challenge conclusions based on empirical data.

For low synergy factors, we have shown that the spontaneous emergence of cyclic dominance between strategies $D, P$, and $B$ is also a possible solution of the system, and indeed it significantly extends the parameter region where the correlation of positive and negative reciprocity is viable. Within the cyclic phase, defectors outperform punishers, punishers outperform the combined strategy, and the combined strategy is able to invade defectors, thereby closing the loop of dominance. In this case, an argument can again be made in favor of the combined strategy over solely rewarding, but since the remaining three strategies become spontaneously entailed in a cycle of dominance, the advantage warranted by the correlation of negative and positive reciprocity is indirect and circumstantial at best. Furthermore, we have demonstrated that the cyclic dominance can terminate in very different ways. Either the average fraction of one strategy vanishes, or, more intriguingly, the amplitude of oscillations diverges in a system-size-independent manner. Thus, although the average fractions of all three strategies are far from zero, the cyclic dominance phase may end abruptly via a discontinuous phase transition. Although phenomena like indirect territorial competition, cyclic dominance, divergent fluctuations of the amplitude of oscillations, as well as previously reported critical phenomena in evolutionary games [64], self-organized adaptation [65,66], and in-group favoritism [67] are all of significant interest to physicists, we emphasize that they would likely require massive efforts to be observed in human experiments. Nevertheless, recent large-scale attempts in this direction promise exciting times ahead $[22,63]$.

Last, it remains to emphasize that punishment is the elementary strategy that is definitively more effective than the combined strategy, while rewarding is not necessarily so. However, rewarding can be made much more potent if rewards are administered not to all cooperators but only to those who themselves reward others. In this case, rewarding can completely outperform punishment at low $\gamma$ and high $\beta$ values, while the situation reverses only if the costs become relatively high compared to the rewards and fines. Yet, in this modified scenario, the act of punishing yields no extra advantages, and in general, strategy $B$ can survive only when strategy $R$ can survive, too. Therefore, even under such altered, rewarding-friendly conditions, there are still no notable evolutionary advantages to be gained by adopting a strategy that combines both positive and negative reciprocity. With this conclusion, we hope that our study will inspire further research aimed at investigating the role of correlated strategies in evolutionary games, and we also hope that more experimental work will be carried out to clarify their role in the evolution of human cooperation.

\section{ACKNOWLEDGMENTS}

This research was supported by the Hungarian National Research Fund (Grant No. K-101490) and the Slovenian Research Agency (Grant No. J1-4055).

[1] M. A. Nowak and R. Highfield, SuperCooperators: Altruism, Evolution, and Why We Need Each Other to Succeed (Free Press, New York, 2011).

[2] C. L. Apicella, F. W. Marlowe, J. H. Fowler, and N. A. Christakis, Social Networks and Cooperation in HunterGatherers, Nature (London) 481, 497 (2012).

[3] E. Fehr and K.M. Schmidt, A Theory of Fairness, Competition, and Cooperation, Q. J. Econ. 114, 817 (1999).

[4] J. Henrich, R. Boyd, S. Bowles, C. Camerer, E. Fehr, H. Gintis, and R. McElreath, In Search of Homo Economicus: Behavioral Experiments in 15 Small-Scale Societies, Am. Econ. Rev. 91, 73 (2001).

[5] E. Fehr and S. Gachter, Cooperation and Punishment in Public Goods Experiments, Am. Econ. Rev. 90, 980 (2000).

[6] J. Henrich, R. McElreath, A. Barr, J. Ensminger, C. Barrett, A. Bolyanatz, J.C. Cardenas, M. Gurven, E. Gwako, N. Henrich, C. Lesorogol, F. Marlowe, D. Tracer, and J. Ziker, Costly Punishment across Human Societies, Science 312, 1767 (2006).

[7] K. Sigmund, Punish or Perish? Retaliation and Collaboration among Humans, Trends Ecol. Evol. 22, 593 (2007).

[8] J. M. Burkart, E. Fehr, C. Efferson, and C. P. van Schaik, Other-Regarding Preferences in a Non-human Primate: Common Marmosets Provision Food Altruistically, Proc. Natl. Acad. Sci. U.S.A. 104, 19762 (2007).

[9] D. Proctor, R. A. Williamson, F. B. M. de Waal, and S. F. Brosnan, Chimpanzees Play the Ultimatum Game, Proc. Natl. Acad. Sci. U.S.A. 110, 2070 (2013).

[10] S. Bowles and H. Gintis, A Cooperative Species: Human Reciprocity and Its Evolution (Princeton University Press, Princeton, NJ, 2011).

[11] S. B. Hrdy, Mothers and Others: The Evolutionary Origins of Mutual Understanding (Harvard University Press, Cambridge, MA, 2011).

[12] G. Szabó and G. Fáth, Evolutionary Games on Graphs, Phys. Rep. 446, 97 (2007).

[13] M. Perc and A. Szolnoki, Coevolutionary Games-A Mini Review, BioSystems 99, 109 (2010).

[14] M. Perc, J. Gómez-Gardeñes, A. Szolnoki, L. M. Floría and Y. Moreno, Evolutionary Dynamics of Group Interactions on Structured Populations: A Review, J. R. Soc. Interface 10, 20120997 (2013).

[15] M. A. Nowak, Evolving Cooperation, J. Theor. Biol. 299, 1 (2012). 
[16] D. G. Rand and M.A. Nowak, Human Cooperation, Trends Cogn. Sci. 17, 413 (2013).

[17] M. A. Nowak, Five Rules for the Evolution of Cooperation, Science 314, 1560 (2006).

[18] M. A. Nowak and R. M. May, Evolutionary Games and Spatial Chaos, Nature (London) 359, 826 (1992).

[19] C. Hauert and M. Doebeli, Spatial Structure Often Inhibits the Evolution of Cooperation in the Snowdrift Game, Nature (London) 428, 643 (2004).

[20] F.C. Santos and J.M. Pacheco, Scale-Free Networks Provide a Unifying Framework for the Emergence of Cooperation, Phys. Rev. Lett. 95, 098104 (2005).

[21] F. Fu, D. I. Rosenbloom, L. Wang, and M. A. Nowak, Imitation Dynamics of Vaccination Behaviour on Social Networks, Proc. R. Soc. B 278, 42 (2011).

[22] C. Gracia-Lázaro, A. Ferrer, G. Ruiz, A. Tarancón, J. A. Cuesta, A. Sánchez, and Y. Moreno, Heterogeneous Networks Do Not Promote Cooperation When Humans Play a Prisoner's Dilemma, Proc. Natl. Acad. Sci. U.S.A. 109, 12922 (2012).

[23] H. Gintis, Strong Reciprocity and Human Sociality, J. Theor. Biol. 206, 169 (2000).

[24] E. Fehr, U. Fischbacher, and S. Gächter, Strong Reciprocity, Human Cooperation and the Enforcement of Social Norms, Human Nature 13, 1 (2002).

[25] R. Boyd, H. Gintis, S. Bowles, and P. J. Richerson, The Evolution of Altruistic Punishment, Proc. Natl. Acad. Sci. U.S.A. 100, 3531 (2003).

[26] S. Bowles and H. Gintis, The Evolution of Strong Reciprocity: Cooperation in Heterogeneous Populations, Theor. Popul. Biol. 65, 17 (2004).

[27] T. Yamagishi, Y. Horita, N. Mifune, H. Hashimoto, Y. Li, M. Shinada, A. Miura, K. Inukai, H. Takagishi, and D. Simunovic, Rejection of Unfair Offers in the Ultimatum Game Is No Evidence of Strong Reciprocity, Proc. Natl. Acad. Sci. U.S.A. 109, 20364 (2012).

[28] B. Egloff, D. Richter, and S.C. Schmukle, Need for Conclusive Evidence That Positive and Negative Reciprocity Are Unrelated, Proc. Natl. Acad. Sci. U.S.A. 110, E786 (2013)

[29] W. Güth, R. Schmittberger, and B. Schwarze, An Experimental Analysis of Ultimatum Bargaining, J. Econ. Behav. Organ. 3, 367 (1982).

[30] D. G. Rand, A. Dreber, T. Ellingsen, D. Fudenberg, and M. A. Nowak, Positive Interactions Promote Public Cooperation, Science 325, 1272 (2009).

[31] D. G. Rand, S. Arbesman, and N. A. Christakis, Dynamic Social Networks Promote Cooperation in Experiments with Humans, Proc. Natl. Acad. Sci. U.S.A. 108, 19193 (2011).

[32] K. Sigmund, E. Fehr, and M. A. Nowak, The Economics of Fair Play, Sci. Am. 286, 82 (2002).

[33] K. Sigmund, Games of Life: Exploration in Ecology, Evolution and Behavior (Oxford University Press, Oxford, England, 1993).

[34] J.W. Weibull, Evolutionary Game Theory (MIT Press, Cambridge, MA, 1995).

[35] J. Hofbauer and K. Sigmund, Evolutionary Games and Population Dynamics (Cambridge University Press, Cambridge, England, 1998).

[36] M.A. Nowak, Evolutionary Dynamics (Harvard University Press, Cambridge, MA, 2006).
[37] K. Sigmund, The Calculus of Selfishness (Princeton University Press, Princeton, NJ, 2010).

[38] K. Binder and D. K. Hermann, Monte Carlo Simulations in Statistical Physics (Springer, Heidelberg, 1988).

[39] T.M. Liggett, Interacting Particle Systems (Springer, New York, 1985).

[40] C. Hilbe and K. Sigmund, Incentives and Opportunism: From the Carrot to the Stick, Proc. R. Soc. B 277, 2427 (2010).

[41] K. Sigmund, H. De Silva, A. Traulsen, and C. Hauert, Social Learning Promotes Institutions for Governing the Commons, Nature (London) 466, 861 (2010).

[42] D. G. Rand and M. A. Nowak, The Evolution of Antisocial Punishment in Optional Public Goods Games, Nat. Commun. 2, 434 (2011).

[43] C. Hilbe and A. Traulsen, Emergence of Responsible Sanctions without Second Order Free Riders, Antisocial Punishment or Spite, Sci. Rep. 2, 458 (2012).

[44] M. Perc and A. Szolnoki, Self-Organization of Punishment in Structured Populations, New J. Phys. 14, 043013 (2012).

[45] A. Szolnoki and M. Perc, Evolutionary Advantages of Adaptive Rewarding, New J. Phys. 14, 093016 (2012).

[46] H. Brandt, C. Hauert, and K. Sigmund, Punishment and Reputation in Spatial Public Goods Games, Proc. R. Soc. B 270, 1099 (2003).

[47] A. Szolnoki, M. Perc, and G. Szabó, TopologyIndependent Impact of Noise on Cooperation in Spatial Public Goods Games, Phys. Rev. E 80, 056109 (2009).

[48] E. Fehr, Don't Lose Your Reputation, Nature (London) 432, 449 (2004).

[49] J. H. Fowler, Second-Order Free-Riding Problem Solved?, Nature (London) 437, E8 (2005).

[50] M. Nakamaru and Y. Iwasa, Evolution of Altruism by Costly Punishment in Lattice-Structured Population: Score-Dependent Viability versus Score-Dependent Fertility, Evol. Ecol. Res. 7, 853 (2005).

[51] D. Helbing, A. Szolnoki, M. Perc, and G. Szabó, Punish, but Not Too Hard: How Costly Punishment Spreads in the Spatial Public Goods Game, New J. Phys. 12, 083005 (2010).

[52] A. Szolnoki and M. Perc, Group-Size Effects on the Evolution of Cooperation in the Spatial Public Goods Game, Phys. Rev. E 84, 047102 (2011).

[53] A. Szolnoki, G. Szabó, and L. Czakó, Competition of Individual and Institutional Punishments in Spatial Public Goods Games, Phys. Rev. E 84, 046106 (2011).

[54] A. Szolnoki, G. Szabó, and M. Perc, Phase Diagrams for the Spatial Public Goods Game with Pool Punishment, Phys. Rev. E 83, 036101 (2011).

[55] D. Helbing, A. Szolnoki, M. Perc, and G. Szabó, Evolutionary Establishment of Moral and Double Moral Standards through Spatial Interactions, PLoS Comput. Biol. 6, e1000758 (2010).

[56] A. Szolnoki and M. Perc, Effectiveness of Conditional Punishment for the Evolution of Public Cooperation, J. Theor. Biol. 325, 34 (2013).

[57] T. Reichenbach, M. Mobilia, and E. Frey, Mobility Promotes and Jeopardizes Biodiversity in 
Rock-Paper-Scissors Games, Nature (London) 448, 1046 (2007).

[58] T. Reichenbach, M. Mobilia, and E. Frey, Noise and Correlations in a Spatial Population Model with Cyclic Competition, Phys. Rev. Lett. 99, 238105 (2007).

[59] M. Mobilia, Oscillatory Dynamics in Rock-Paper-Scissors Games with Mutations, J. Theor. Biol. 264, 1 (2010).

[60] M. Mobilia, Fixation and Polarization in a Three-Species Opinion Dynamics Model, Europhys. Lett. 95, 50002 (2011).

[61] Z.-X. Wu, X.-J. Xu, Y. Chen, and Y.-H. Wang, Spatial Prisoner's Dilemma Game with Volunteering in NewmanWatts Small-World Networks, Phys. Rev. E 71, 037103 (2005).

[62] A. Szolnoki, M. Perc, and G. Szabó, Defense Mechanisms of Empathetic Players in the Spatial Ultimatum Game, Phys. Rev. Lett. 109, 078701 (2012).
[63] C. Gracia-Lázaro, J.A. Cuesta, A. Sánchez, and Y. Moreno, Human Behavior in Prisoner's Dilemma Experiments Suppresses Network Reciprocity, Sci. Rep. 2, 325 (2012).

[64] D. G. Hernández and D. H. Zanette, Critical Transition Induced by Neighbourhood Size in Evolutionary Spatial Games, Eur. Phys. J. B 82, 361 (2011).

[65] S. Steingrube, M. Timme, F. Wörgötter, and P. Manoonpong, Self-Organized Adaptation of a Simple Neural Circuit Enables Complex Robot Behaviour, Nat. Phys. 6, 224 (2010).

[66] S. Lee, P. Holme, and Z.-X. Wu, Emergent Hierarchical Structures in Multiadaptive Games, Phys. Rev. Lett. 106, 028702 (2011).

[67] F. Fu, C. E. Tarnita, N. A. Christakis, L. Wang, D. G. Rand, and M. A. Nowak, Evolution of In-Group Favoritism, Sci. Rep. 2, 460 (2012). 